\title{
ANÁLISE MULTIVARIADA DE ATRIBUTOS MICROBIOLÓGICOS E QUÍMICOS DO SOLO EM FLORESTAS COM Araucaria angustifolia $^{(1)}$
}

\author{
Dilmar Baretta $^{(2)}$, Carolina Riviera Duarte Maluche Baretta ${ }^{(3)} \&$ \\ Elke Jurandy Bran Nogueira Cardoso ${ }^{(4)}$
}

\begin{abstract}
RESUMO
Araucaria angustifolia é uma espécie de árvore ameaçada de extinção no Brasil e pouco se conhece sobre os atributos edáficos dessas florestas. Este estudo foi realizado com o objetivo de identificar diferenças entre áreas com araucária naturais e reflorestadas, com base em atributos microbiológicos e químicos do solo, por meio de métodos multivariados, como a análise canônica discriminante (ACD) e a análise de correlação canônica (ACC). As áreas estudadas incluem: 1 . floresta nativa com araucária $(\mathrm{NF}) ; 2$. reflorestamento de araucária $(\mathrm{R}) ; 3$. reflorestamento de araucária submetido a incêndio acidental (RF); e 4. pastagem natural com araucárias nativas e ocorrência de incêndio (NPF). Foram selecionadas, ao acaso, quinze árvores de araucária por área e sob a copa de cada uma delas foram retiradas três amostras de solo, em três épocas contrastantes. A ACD foi aplicada aos atributos microbianos: $\mathrm{C}$ da biomassa microbiana (CBM), respiração basal $\left(\mathrm{C}-\mathrm{CO}_{2}\right)$ e quociente metabólico $\left(q \mathrm{CO}_{2}\right)$, enquanto a $\mathrm{ACC}$ foi aplicada aos atributos microbianos e químicos do solo [pH $\left(\mathrm{CaCl}_{2}\right), \mathrm{C}$ orgânico total (COT) e teores de $\mathrm{P}, \mathrm{K}, \mathrm{Ca}, \mathrm{Mg}$ e $(\mathrm{H}+\mathrm{Al})$. Os atributos microbianos e químicos do solo apresentaram alta correlação canônica, com destaque para o CBM e C-CO $\mathrm{CO}_{2}$, entre os atributos microbianos, e para COT e $\mathrm{P}$, entre os atributos químicos do solo. A ACC mostrou que 52 \% da variação total dos atributos microbianos é explicada pela variação dos atributos químicos, e $36 \%$ da variação total dos atributos químicos é explicada pelos atributos microbianos do solo das áreas estudadas. A ACD identificou que a contribuição de cada atributo
\end{abstract}

\footnotetext{
(1) Parte da Tese de Doutorado do primeiro autor, apresentada ao Programa de Pós-graduação em Solos e Nutrição de Plantas da Escola Superior de Agricultura Luiz de Queiroz - ESALQ/USP. Trabalho apresentado no XXXI Congresso Brasileiro de Ciência do Solo, 2007 (Gramado, RS).

(2) Professor do Departamento de Zootecnia, Centro de Educação Superior do Oeste - CEO/UDESC. Laboratório de Solos, Rua Benjamin Constant, 84-E, CEP 89806-070, Chapecó (SC). E-mail: dilmarbaretta@gmail.com

(3) Recém-Doutora pelo Programa de Pós-graduação em Solos e Nutrição de Plantas, Escola Superior de Agricultura "Luiz de Queiroz" - ESALQ/USP. Av. Pádua Dias, 11, Caixa Postal 9, CEP 13418-900, Piracicaba (SP). E-mail: cmaluche@esalq.usp.br

(4) Professora Titular do Departamento de Ciência do Solo, ESALQ/USP. E-mail: ejbncard@esalq.usp.br
} 
microbiano para a separação das áreas sofreu variação ao longo do tempo, e discriminou o CBM como sendo o atributo microbiano mais importante, seguido pelo $\mathrm{C}_{-} \mathrm{CO}_{2}$. ACC e ACD são importantes ferramentas no estudo de indicadores de qualidade do solo.

Termos de indexação: análise de correlação canônica, análise canônica discriminante, bioindicador de qualidade, carbono da biomassa microbiana.

\title{
SUMMARY: MULTIVARIATE ANALYSIS OF SOIL MICROBIOLOGICAL AND CHEMICAL ATTRIBUTES IN FORESTS WITH ARAUCARIA ANGUSTIFOLIA
}

\begin{abstract}
Araucaria angustifolia is an endangered tree species in Brazil and little is known about the soil attributes of these pine forests. This study was carried out to identify differences between natural and reforested Araucaria areas, in terms of soil microbiological and chemical attributes, with multivariate canonical discriminant analysis $(C D A)$ and canonical correlation analysis (CCA). The studied areas included: 1. native forest with Araucaria (NF); 2. Araucaria reforestation ( $R$ ); 3. Araucaria reforestation burnt by an accidental fire (RF); and 4. native grass pasture with native Araucaria and burnt by an intense accidental fire (NPF). In each area, 15 pine trees were selected and three soil samples collected from under each tree crown, in three different seasons. The CDA was applied to the soil microbiological attributes: carbon of microbial biomass $(\mathrm{CMB})$, basal respiration $\left(\mathrm{C}-\mathrm{CO}_{2}\right)$ and metabolic quotient $\left(\mathrm{qCO}_{2}\right)$, while $C C A$ was performed with the soil microbiological and soil chemical attributes $\left[\mathrm{pH}\left(\mathrm{CaCl}_{2}\right)\right.$, total organic carbon (TOC), and the contents of $\mathrm{P}, \mathrm{K}, \mathrm{Ca}, \mathrm{Mg}$, and $\mathrm{H}+\mathrm{All}$. The canonical correlation between soil microbiological and chemical attributes was highly significant, with a higher contribution of $\mathrm{CMB}$ and $\mathrm{C}-\mathrm{CO}_{2}$ to the microbiological attributes, and of TOC and $\mathrm{P}$ to the chemical attributes. The CCA indicated that the variation of the chemical attributes can explain $52 \%$ of the total data variability of the microbiological attributes, and the soil microbiological attributes of the areas $36 \%$ of the total data variability of the chemical attributes. The CDA suggested that the contribution of each microbiological attribute to the discrimination of the areas was season-dependent, and discriminated CMB as the most important microbiological indicator, followed by $C-\mathrm{CO}_{2}$. The tools $C C A$ and $C D A$ proved to be essential in the study of soil quality indicators.
\end{abstract}

Index terms: canonical correlation analysis, canonical discriminant analysis, soil quality bioindicator, carbon of the microbial biomass.

\section{INTRODUÇÃO}

A Floresta Ombrófila Mista (Floresta de Araucária) no Brasil tem como principal representante a Araucaria angustifolia (Bert.) O. Kuntze, com uma ampla distribuição geográfica, pois engloba os Estados do Paraná, Rio Grande do Sul e Santa Catarina, com manchas esparsas no Estado de São Paulo (Huech, 1972), sul de Minas Gerais e sudoeste do Rio de Janeiro (Reitz et al., 1988). Entretanto, a fragmentação que esta floresta vem sofrendo nos últimos anos, provocada pela forte interferência antrópica, é um dos grandes problemas atuais, o que levou o IBAMA a classificá-la como espécie da flora brasileira ameaçada de extinção (IBAMA, 1992).

A intervenção antrópica em florestas com araucárias naturais e reflorestadas na região de
Campos do Jordão, SP, pode causar mais perdas do que ganhos de $\mathrm{C}$, provocando perda da qualidade do solo, especialmente dos atributos microbianos ao longo do tempo. Tais perdas são devidas à liberação de $\mathrm{CO}_{2}$ pela respiração basal durante o processo de decomposição da matéria orgânica do solo (MOS) e respiração de raízes e animais. Como a degradação da MOS é realizada por microrganismos heterotróficos, a atividade microbiana do solo pode ser utilizada para entender os processos de mineralização e intensidade dos fluxos de energia no solo (Alef \& Nannipieri, 1995; Choromanska \& Deluca, 2001).

A quantificação do $\mathrm{C}$ da biomassa microbiana $(\mathrm{CBM})$, respiração basal $\left(\mathrm{C}-\mathrm{CO}_{2}\right)$ e suas relações como, por exemplo, quociente metabólico $\left(q \mathrm{CO}_{2}\right)$, tem sido utilizada para estudar os processos de ciclagem e transformação de nutrientes (Maluche-Baretta et al., 2006), bem como para avaliar a dinâmica da matéria 
orgânica do solo (Gama-Rodrigues, 1999; Anderson, 2003). Além de armazenador de nutrientes, o CBM pode servir como indicador rápido da sensibilidade da microbiota às interferências nos ecossistemas (Dumontet et al., 1996; Neary et al., 1999; Choromanska \& Deluca, 2001). Todavia, os trabalhos que visam a avaliar o efeito da intervenção antrópica sobre os atributos microbianos do solo em Florestas de Araucária são bastante escassos, sendo praticamente inexistentes os dados de literatura que procuram estabelecer relações entre os atributos químicos e microbianos do solo, por meio de métodos multi-variados, como a análise canônica discriminante (ACD) e análise de correlação canônica (ACC).

Embora os estudos que envolvem os atributos microbianos e químicos do solo tenham aumentado muito nas últimas décadas, dado o crescente interesse na compreensão dos processos que ocorrem nos agroecossistemas (Gelsomino et al., 2006; Mariani et al., 2006; Franchini et al., 2007), ainda existem problemas nas análises estatísticas dos dados químicos e biológicos do solo por superar (Baretta et al., 2005). O modelo de análise estatística univariada convencional torna-se menos sensível em sistemas biológicos, em razão das particularidades de cada manejo (Maluche-Baretta et al., 2006), tratando somente uma das diversas variáveis a cada tempo, e não considera o efeito conjunto dos inúmeros atributos químicos e microbianos avaliados. Assim, a necessidade de análise multivariada surge toda vez que o pesquisador tem vários atributos a serem analisados e necessita estudar simultaneamente suas relações (Gauch Jr., 1982; Gelsomino et al., 2006; Mariani et al., 2006).

A técnica multivariada, conhecida como ACD, é uma ferramenta estatística, que permite estabelecer não só a identificação de diferenças entre tratamentos, mas também qual é o atributo que mais contribui para a separação entre as áreas estudadas (CruzCastillo et al., 1994; Baretta et al., 2005), permitindo, assim, a eliminação de tempo e dinheiro gastos com aqueles atributos do solo que expressam baixa resposta (Baretta et al., 2005). Essa abordagem é muito útil porque, além de identificar os atributos químicos ou microbianos do solo mais relevantes para a separação das áreas, permite considerá-lo como um indicador sensível para avaliação da intervenção antrópica (Choromanska \& Deluca, 2001; Gelsomino et al., 2006; Mariani et al., 2006; Franchini et al., 2007), bem como do status da sustentabilidade das Florestas de Araucária.

O estudo das relações entre os atributos microbianos e químicos do solo obtido pela ACC, por sua vez, pode auxiliar no entendimento do funcionamento do solo nas Florestas de Araucária, bem como da qualidade do solo (Baretta et al., 2005; Gelsomino et al., 2006; Mariani et al., 2006). Diante desse contexto, este estudo pretendeu identificar diferenças entre florestas com araucária naturais e reflorestadas, impactadas ou não pela queima acidental, com base em atributos microbianos e químicos do solo, por meio de métodos multivariados, como a análise canônica discriminante (ACD) e a análise de correlação canônica (ACC).

\section{MATERIAL E MÉTODOS}

O estudo foi realizado no município de Campos do Jordão, SP, no período de setembro de 2004 a agosto de 2005, em quatro áreas representativas de florestas de $A$. angustifolia, dentro do Parque Estadual de Campos do Jordão, distante $210 \mathrm{~km}$ da cidade de São Paulo, e situado a $22^{\circ} 39^{\prime}$ latitude Sul e $45^{\circ} 27^{\prime}$ longitude Oeste, com altitude média de $1519 \mathrm{~m}$ acima do nível do mar. O relevo é suave ondulado, com declividade média de $0,1702 \mathrm{~m} \mathrm{~m}^{-1}$. O solo é classificado como Latossolo Vermelho-Amarelo distrófico (Embrapa, 1999). Maiores informações sobre as características químicas do solo podem ser obtidas em Baretta et al. (2007). O clima da região é subtropical de altitude, mesotérmico e úmido ( $\mathrm{Cfb}$, conforme a classificação de Köppen). A maior precipitação pluviométrica concentra-se no verão (dezembro a fevereiro) observando-se, no mês de janeiro de 2005, mais de $230 \mathrm{~mm}$ e em agosto de 2005 , somente $6 \mathrm{~mm}$ (deficiência hídrica). O mês mais frio é julho, com temperatura média mensal próxima a $11,5^{\circ} \mathrm{C}$ e os meses mais quentes janeiro e fevereiro, com temperatura média superior a $17,0^{\circ} \mathrm{C}$ (Baretta et al., 2007).

As áreas com araucárias selecionadas foram: 1. floresta nativa com predominância de araucária, em clímax, com baixa interferência antrópica (NF); 2. reflorestamento de araucária, plantado em 1959 (R); 3. reflorestamento de araucária, plantado em 1959, submetido a um incêndio acidental intenso em julho de 2001 (RF), e 4. pastagem natural com araucárias nativas (NPF), submetida a incêndio acidental intenso em setembro de 2004. Todas as áreas estão localizadas em toposseqüência e altitudes semelhantes. Considerando o pouco conhecimento sobre a variabilidade dos atributos do solo nas áreas estudadas, reduziu-se a extensão de cada parcela (aproximadamente $0.3 \mathrm{ha}$ ), visando a controlar algumas características críticas do local e a evitar os efeitos de variáveis não-controláveis. Maiores informações sobre a flora arbórea, arbustiva e herbácea do Parque Estadual de Campos do Jordão e das áreas estudadas podem ser obtidas em Robim et al. (1990) e Baretta (2007).

Para avaliar os atributos microbianos do solo, foram realizadas coletas em setembro de 2004 ( $2 \mathrm{~h}$ após a queima na área NPF), fevereiro de 2005 e agosto de 2005. Foram escolhidas, ao acaso, quinze árvores de araucária por área e sob a copa de cada uma delas foram retiradas três subamostras de solo na profundidade de $0-20 \mathrm{~cm}$. Posteriormente, as amostras foram acondicionadas em sacos plásticos e transportadas em caixas de isopor com gelo para o 
laboratório, onde foram mantidas em câmara fria até serem analisadas. Para as análises, as amostras foram homogeneizadas e passadas em peneira com malha de $2 \mathrm{~mm}$. Uma subamostra foi retirada (somente em setembro de 2004), para determinação dos seguintes atributos químicos do solo: $\mathrm{pH}$ em $\left(\mathrm{CaCl}_{2}\right)$, matéria orgânica (Método Walkey-Black), teores de $\mathrm{P}$ (Resina), de $\mathrm{K}$, de $(\mathrm{H}+\mathrm{Al})$, de $\mathrm{Ca}$ e $\mathrm{Mg}$ trocáveis, conforme método descrito em Raij et al. (2001).

O C da biomassa microbiana (CBM) foi determinado pelo método da fumigação-extração (Vance et al., 1987). O C orgânico total (COT) do solo foi estimado por oxidação úmida com $\mathrm{K}_{2} \mathrm{Cr}_{2} \mathrm{O}_{4} \mathrm{e}$ $\mathrm{H}_{2} \mathrm{SO}_{4}$, conforme proposto por Raij et al. (2001). A partir dos resultados de CBM e COT, foi calculada a relação entre as duas características, expressa como a percentagem de $\mathrm{C}$ microbiano em relação ao $\mathrm{C}$ total do solo (Anderson, 1994). A atividade microbiana foi avaliada pela determinação da respiração basal (C$\mathrm{CO}_{2}$ ) em $50 \mathrm{~g}$ de amostra de solo, pelo método descrito por Alef \& Nannipieri (1995), por meio da quantificação do $\mathrm{CO}_{2}$ liberado no processo de respiração microbiana durante treze dias de incubação $\left(28^{\circ} \mathrm{C}\right)$. Utilizaram-se os resultados de C$\mathrm{CO}_{2}$ e do CBM para calcular o quociente metabólico $\left(q \mathrm{CO}_{2}\right)$, que representa a quantidade de $\mathrm{C}-\mathrm{CO}_{2}$ liberada em determinado tempo, por unidade de $\mathrm{C}$ microbiano (mg C- $\mathrm{CO}_{2} / \mathrm{g} \mathrm{C} \mathrm{mic} \mathrm{h}^{-1}$ ), conforme proposto por Anderson \& Domsch (1993).

Os atributos microbianos foram submetidos à análise canônica discriminante (ACD) para identificar quais deles foram mais relevantes na separação das áreas amostradas (Cruz-Castilho et al. 1994; Baretta et al., 2005; Maluche-Baretta et al., 2006). Quando verificada diferença significativa entre as áreas, pela ACD, foi efetuado teste de comparação de médias nos valores dos coeficientes canônicos padronizados (CCP), nas distintas funções canônicas, por meio do teste de LSD $(\mathrm{P}<0,05)$, conforme Cruz-Castilho et al. (1994). Também foram feitas análises de correlação canônica (ACC) entre os atributos microbianos (CBM, C- $\mathrm{CO}_{2}$ e $\left.q \mathrm{CO}_{2}\right)$ e químicos do solo $\left[\mathrm{pH}\left(\mathrm{CaCl}_{2}\right)\right.$ COT e teores de P, K, Ca, Mg e (H+Al)]. As análises multivariadas foram feitas com o SAS versão 8.2 (SAS, 2002).

\section{RESULTADOS E DISCUSSÃO}

\section{Análise canônica discriminante (ACD)}

O modelo utilizado na ACD para avaliar as modificações nos atributos microbianos do solo explicou boa parte da variabilidade biológica nas áreas estudadas, uma vez que a primeira e segunda função canônica discriminante $\left(\mathrm{FCD}_{1}\right.$ e $\left.\mathrm{FCD}_{2}\right)$ apresentaram correlações canônicas de 93 e 65 \% na amostragem de setembro, de 82 e $43 \%$, em fevereiro, e de 68 e $34 \%$, em agosto, respectivamente; sendo as duas funções ajustáveis para explicar as variações encontradas para os valores de $\mathrm{COT}, \mathrm{CBM}, \mathrm{C}-\mathrm{CO}_{2}$ e $q \mathrm{CO}_{2}$. A relação CBM:COT é uma relação de divisão direta e altamente influenciada pelos valores de CBM, o que diminui a confiabilidade dessa análise multivariada pelo efeito da colinearidade dos dados (Cruz-Castillo et al., 1994; Baretta et al., 2005). Assim, essa relação microbiana não foi apresentada neste estudo.

O teste LSD a $5 \%$ da média de todos os coeficientes canônicos padronizados (CCP) mostrou dentro da $\mathrm{FCD}_{1}$ que todas as áreas com araucárias estudadas são diferentes entre si, sendo a magnitude dessas diferenças dependente das épocas de amostragem (Quadro 1). Na amostragem de setembro (2004), dentro da $\mathrm{FCD}_{1}$, a Floresta de Araucária natural (NF) obteve a maior média, seguida do reflorestamento introduzido $(\mathrm{R})$ e impactado $(\mathrm{RF})$ pela queima, que não diferiram entre si, e pela área de pastagem com araucária impactada pela queima acidental (NPF), respectivamente (Quadro 1). A $\mathrm{FCD}_{2}$, como explicou pouco (menos de $13 \%$ ) da variação total dos dados microbianos nas três épocas de amostragens (setembro, fevereiro e agosto), não será discutida. Já em fevereiro (2005), as áreas NF e RF obtiveram as maiores médias, seguida da área $\mathrm{R}$, que não diferiu da área RF, e NPF, respectivamente. E, finalmente, em agosto (2005), as áreas NF, R e RF obtiveram as maiores médias, seguidas da área NPF. Baretta et al. (2005), estudando o efeito da queima da pastagem nativa sobre atributos microbianos em Lages, SC, observaram essa mesma tendência, com menores valores médios de CCP na área de campo nativo submetido à queima, em comparação com as áreas de campo nativo não queimado nos últimos 50 anos e floresta nativa de araucária.

Quadro 1. Análise da variância média dos coeficientes canônicos padronizados (CCP) da primeira função canônica discriminante $\left(\mathrm{FCD}_{1}\right)$ referentes aos valores de atributos microbianos do solo, discriminando as Florestas de Araucária natural (NF), introduzida (R), introduzida impactada (RF) e área de pastagem natural com araucárias nativas impactadas pela queima acidental (NPF), em setembro de 2004, fevereiro de 2005 e agosto de $2005(n=15)$

\begin{tabular}{lccccc}
\hline \multicolumn{1}{c}{ Época } & NF & R & RF & NPF & CV(\%) \\
\hline Setembro de 2004 & $2,63 \mathrm{a}$ & $0,53 \mathrm{~b}$ & $0,53 \mathrm{~b}$ & $-4,11 \mathrm{c}$ & 1,11 \\
Fevereiro de 2005 & $1,21 \mathrm{a}$ & $0,21 \mathrm{~b}$ & $1,12 \mathrm{ab}$ & $-3,17 \mathrm{c}$ & 1,41 \\
Agosto de 2005 & $0,63 \mathrm{a}$ & $0,70 \mathrm{a}$ & $0,82 \mathrm{a}$ & $-1,79 \mathrm{~b}$ & 3,64 \\
\hline
\end{tabular}

Médias seguidas da mesma letra minúscula, nas linhas, não diferem entre si a $5 \%$ pelo teste $\mathrm{LSD} .{ }^{(1)} \mathrm{CV}$ : Coeficiente de variação. 
A contribuição individual de cada atributo microbiano para a separação das áreas com araucária (Figura 1) é expressa pelos coeficientes da taxa de discriminação paralela (TDP), que resulta do produto entre os coeficientes canônicos padronizados (CCP) e de correlação $(r)$. Os valores $r$ refletem informações univariadas e mostram a contribuição individual de cada atributo estudado na separação das áreas estudadas (Quadro 2). O TDP, por sua vez, apresenta valores relativos à contribuição conjunta de $r \mathrm{eCCP}$ para a distinção das áreas $(\mathrm{TDP}=r \times \mathrm{CCP})$. Estudos recentes indicam que o TDP é mais recomendado quando o pesquisador deseja discriminar áreas (CruzCastillo et al., 1994; Baretta et al., 2005; MalucheBaretta et al., 2006). Assim, evidenciou-se, por meio dos valores de TDP, que os atributos microbianos do solo foram eficientes para separar as áreas amostradas, com potencial como indicadores sensíveis, independentemente da época de amostragem. Entretanto, para cada época de amostragem, a contribuição de cada atributo microbiano para separar as áreas foi diferenciada (Quadro 2).

$\mathrm{Na}$ amostragem de setembro (2004), dentro da $\mathrm{FCD}_{1}$, os atributos microbianos CBM e C- $\mathrm{CO}_{2}$ apresentaram os maiores valores positivos de coeficientes TDP, correspondentes a 0,35 e 0,28 (Quadro 2), o que demonstra que o maior grau de dissimilaridade (separação) das áreas é explicado, principalmente, por estes atributos microbianos. Todavia, os atributos $q \mathrm{CO}_{2}$ e COT, apesar de apresentarem valores mais baixos $(0,17$ e 0,19 , respectivamente), também foram importantes para separar as áreas estudadas.

$\mathrm{Na}$ amostragem de fevereiro (2005), o comportamento foi semelhante ao da amostragem de setembro (2004), ou seja, dentro da $\mathrm{FCD}_{1}$, novamente o C-CO ${ }_{2}$ e o CBM foram os atributos microbianos que apresentaram maiores valores de TDP $(0,45$ e 0,32, respectivamente), e os mais relevantes na separação entre as áreas estudadas (Quadro 2). Já os atributos $q \mathrm{CO}_{2}$ e o COT apresentaram valores de coeficiente TDP baixo $(0,23$ e $-0,00$, respectivamente), contribuindo menos $\left(q \mathrm{CO}_{2}\right)$ ou nada (COT) na função indicada para a separação entre as áreas amostradas (Quadro 2). Por outro lado, na coleta de agosto (2005), na FCD 1 , o CBM foi o atributo que mais contribuiu para a separação das áreas, com valor de coeficiente TDP de 0,72, sendo responsável por praticamente toda a separação das áreas. Estudos recentes têm reportado o CBM como sendo o atributo microbiano mais sensível para discriminar tratamentos (Baretta et al., 2005; MalucheBaretta et al., 2006); entretanto, ainda há necessidade de maiores pesquisas neste sentido.

O teste multivariado de Wilk's Lambda indicou haver diferença altamente significativa $(p<0,0001)$ entre as quatro áreas com araucárias e entre as três épocas de amostragem (Figura 1), por isso realizou-se uma ACD para cada época de amostragem. Da ACD resultaram valores do coeficiente canônico padronizado
(CCP). Tal coeficiente mostrou simultaneamente o comportamento multivariado das variáveis microbianas estudadas para promover a separação entre as áreas, em resposta ao estudo de variáveis independentes múltiplas, analisadas simultaneamente. No presente estudo, a $\mathrm{FCD}_{1}$ explicou a maior parte da variação total em setembro (89 \%), fevereiro (90 \%) e agosto (81\%), enquanto a $\mathrm{FCD}_{2}$, apenas 10,9 e $12 \%$, em setembro, fevereiro e agosto, respectivamente (Figura 1).

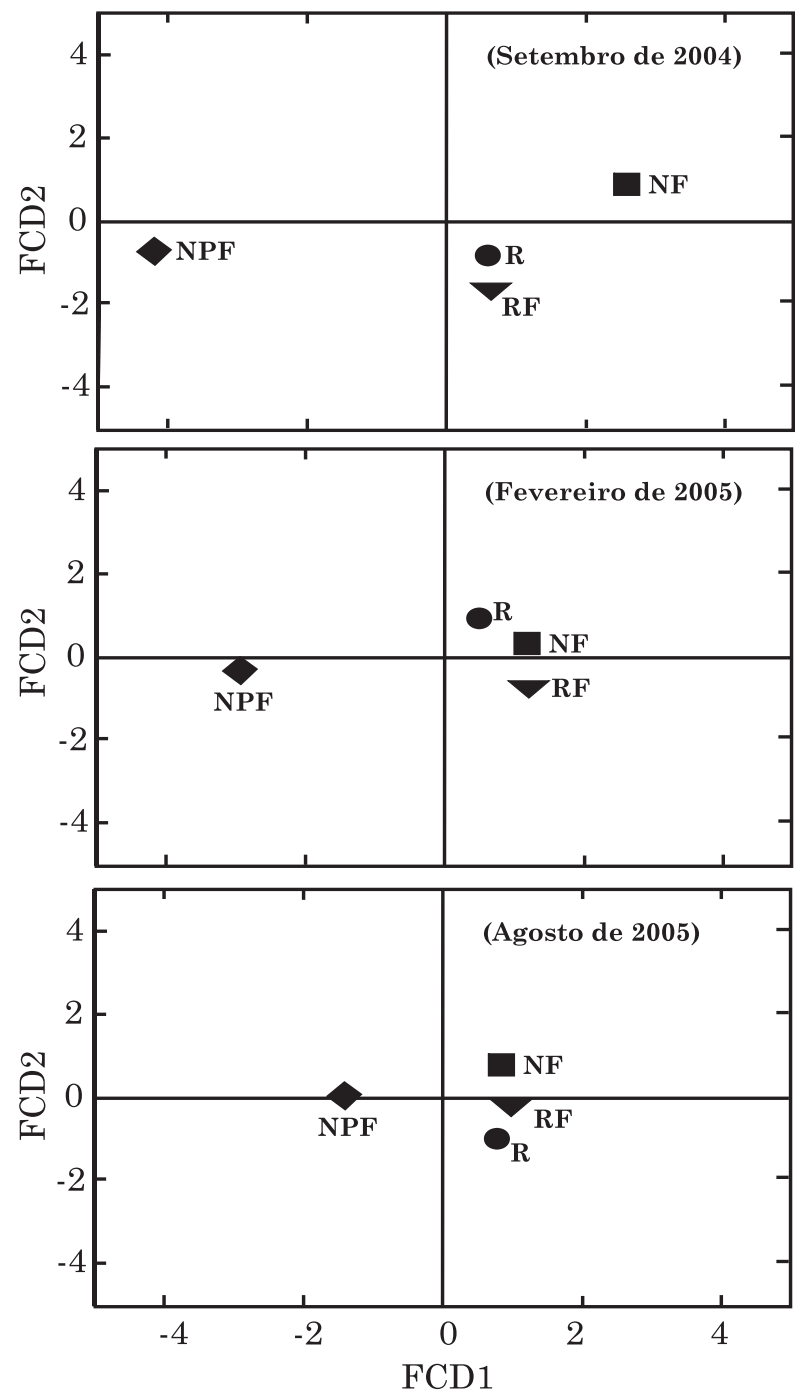

Figura 1. Relação entre a primeira e segunda função canônica discriminante $\left(\mathrm{FCD}_{1}\right.$ e $\left.\mathrm{FCD}_{2}\right)$ sobre as médias (centróides) dos coeficientes canônicos padronizados (CCP) referentes aos atributos carbono da biomassa microbiana (CBM), respiração basal (C-CO $\left.\mathrm{CO}_{2}\right)$, quociente metabólico $\left(q \mathrm{CO}_{2}\right)$ e carbono orgânico total (COT) do solo, discriminando as Florestas de Araucária natural (NF), introduzida (R), introduzida impactada (RF) e área de pastagem natural com araucárias nativas impactadas pela queima acidental (NPF), em setembro de 2004, fevereiro de 2005 e agosto de $2005(n=15)$. 
Quadro 2. Valores de coeficiente de correlação canônica (r), coeficiente canônico padronizado (CCP) e coeficiente da taxa de discriminação paralela (TDP) dentro da primeira função canônica discriminante $\left(\mathrm{FCD}_{1}\right)$ referentes aos atributos carbono da biomassa microbiana $(\mathrm{CBM})$, respiração basal $\left(\mathrm{C}-\mathrm{CO}_{2}\right)$, quociente metabólico $\left(q \mathrm{CO}_{2}\right)$ e carbono orgânico total (COT), nas quatro áreas com araucárias estudadas, independentemente do tratamento

\begin{tabular}{lccc}
\hline Atributo & $\boldsymbol{r}$ & $\mathbf{C C P}$ & TDP \\
\hline & & Setembro de 2004 & 0,3508 \\
$\mathrm{CBM}$ & 0,6571 & 0,5339 & 0,2805 \\
$\mathrm{C}-\mathrm{CO}_{2}$ & 0,4986 & 0,5625 & 0,1726 \\
$\boldsymbol{q C O} 2$ & $-0,5264$ & $-0,3280$ & 0,1959 \\
$\mathrm{COT}$ & 0,4464 & 0,4388 & 0,3174 \\
& & Fevereiro de 2005 & 0,4548 \\
$\mathrm{CBM}$ & $-0,4972$ & $-0,6384$ & 0,2276 \\
$\mathrm{C}-\mathrm{CO} 2$ & 0,8882 & 0,5121 & $-0,0000$ \\
$\boldsymbol{q C O} 2$ & 0,3052 & 0,7458 & 0,7252 \\
$\mathrm{COT}$ & 0,0000 & $-0,0473$ & 0,0560 \\
& & Agosto de 2005 & 0,2187 \\
$\mathrm{CBM}$ & $-0,7864$ & $-0,9222$ & 0,0000 \\
\hline
\end{tabular}

Pode-se observar em setembro (2004) que a $\mathrm{FCD}_{1}$ separa a área NF, com maiores valores de CCP, das áreas $\mathrm{R}$ e RF, enquanto a área NPF ficou afastada das demais, com menores valores (negativos) de coeficiente CCP (Figura 1). Já nas amostragens de fevereiro e agosto de 2005 , dentro da $\mathrm{FCD}_{1}$, pode-se visualizar que a FCD1 separa as florestas NF, R e RF da área NPF. A diferença em termos de proximidade dos valores médios de CCP (centróides) da área NPF em relação às demais áreas estudadas diminuiu com o tempo após a queima acidental, de setembro de 2004 para agosto de 2005 (Figura 1). Tais variações sazonais nos atributos microbianos estão relacionadas, dentre outros fatores, com as condições edafoclimáticas, histórico das áreas, especialmente do nível de intervenção antrópica e da qualidade dos resíduos vegetais aportados (Wardle \& Hungria, 1994; Tiedje et al., 2001; Baretta et al., 2005).

\section{Análise de correlação canônica (ACC)}

Na ACC, apenas três correlações canônicas foram estudadas entre os atributos microbianos (CBM, C$\left.\mathrm{CO}_{2}, q \mathrm{CO}_{2}\right)$ e químicos do solo: $\mathrm{pH}\left(\mathrm{CaCl}_{2}\right)$, COT, e teores de $\mathrm{P}, \mathrm{K}, \mathrm{Ca}, \mathrm{Mg}$ e $(\mathrm{H}+\mathrm{Al})$, o que foi limitado pelo número de atributos microbianos. A relação CBM:COT e a variável matéria orgânica do solo são relações de divisão direta e altamente influenciada pelos valores de CBM e de COT, por isso foram retiradas da ACC, aumentando assim a confiabilidade dos dados apresentados. A primeira correlação canônica entre atributos químicos e microbianos foi de 0,84 $(p<0,0001)$, sendo $62 \%$ da variação dos escores da primeira variável canônica dos atributos microbianos explicada pelos escores da primeira variável canônica dos atributos químicos (Figura 2). A segunda e a terceira correlação canônica não foram significativas, assim não foram consideradas no presente estudo. Dessa forma, apenas a primeira correlação canônica dos dados será analisada e discutida, considerando o seu maior valor e nível de significância em relação às outras correlações canônicas.

A variável canônica biológica 1 (VCB1) apresentou maiores valores de coeficientes canônicos padronizados (CCP) e de correlação canônica (CC) para o atributo microbiano CBM (0,36 e 0,87, respectivamente), seguido do $\mathrm{C}-\mathrm{CO}_{2}(0,18$ e 0,79 , respectivamente) (Quadro 3). Os sinais positivos de $\mathrm{CBM}$ e $\mathrm{C}-\mathrm{CO}_{2}$ indicam que as áreas apresentaram um elevado valor de CBM e C-CO $\mathrm{CO}_{2}$, sendo atributos com alto potencial para discriminação, conforme apresentado no quadro $2 . \mathrm{O} q \mathrm{CO}_{2}$ apresentou valor negativo para o CCP e CC (-0,60 e -0,93, respectivamente), sendo, portanto, considerado atributo supressor e com pouca influência na discriminação das áreas. Todavia, o $q \mathrm{CO}_{2}$ tem sido reportado como sendo um indicador da condição de estresse da biomassa microbiana do solo (Baretta et al., 2005; Maluche-Baretta et al., 2006). 


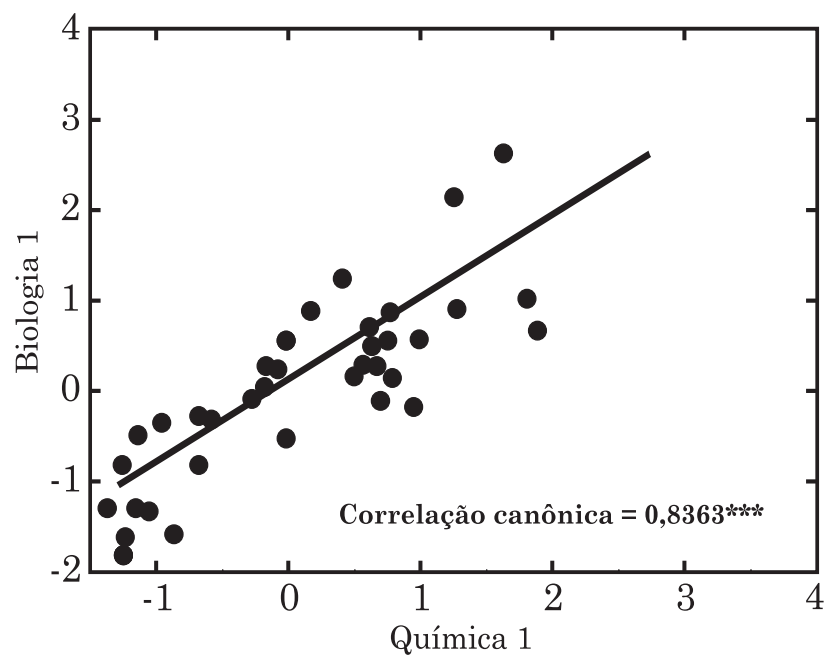

Figura 2. Análise de Correlação Canônica (ACC) entre atributos microbianos (C-CO $\mathrm{CO}_{2}, \mathrm{CBM}$ e $\left.q \mathrm{CO}_{2}\right)$ e químicos do solo [pH $\left(\mathrm{CaCl}_{2}\right)$, COT e teores de $\mathrm{P}$, $\mathrm{K}, \mathrm{Ca}, \mathrm{Mg}$ e $(\mathrm{H}+\mathrm{Al})]$ em Florestas de Araucária natural (NF), introduzida (R), introduzida impactada (RF) e área de pastagem natural com araucárias nativas impactadas pela queima acidental (NPF), independentemente do tratamento $(n=15)$.

Quadro 3. Coeficientes canônicos padronizados (CCP) e correlação canônica (CC) quanto aos atributos microbianos das variáveis canônicas biológicas 1 (VCB1) e químicas 1 (VCQ1) do solo, independentemente do tratamento $(n=15)$.

\begin{tabular}{lccc}
\hline \multicolumn{1}{c}{ Atributo } & $\mathbf{C C P}$ & $\mathbf{C C}$ \\
\hline & & $\mathrm{VCB} 1$ \\
$\mathrm{CBM}$ & 0,36 & & 0,87 \\
$\mathrm{C}^{-} \mathrm{CO}_{2}$ & 0,18 & & 0,79 \\
$\mathbf{q C O} 2$ & $-0,60$ & & $-0,93$ \\
& & $\mathrm{VCQ} 1$ & \\
$\mathrm{pH}\left(\mathrm{CaCl}_{2}\right)$ & $-0,26$ & & $-0,57$ \\
$\mathrm{COT}$ & 1,03 & & 0,78 \\
$\mathrm{P}$ & 0,46 & & 0,74 \\
$\mathrm{~K}$ & $-0,23$ & & 0,03 \\
$\mathrm{Ca}$ & 0,52 & 0,33 \\
$\mathrm{Mg}$ & $-0,69$ & 0,12 \\
$\mathrm{H}+\mathrm{Al}$ & $-0,53$ & 0,71 \\
\hline
\end{tabular}

Na variável química 1 (VCQ1), os atributos COT, $\mathrm{Ca}$ e $\mathrm{P}$ apresentaram valores altos de $\mathrm{CCP}(1,03,0,52$ e 0,46 , respectivamente), indicando que esses são diretamente relacionados com os atributos microbianos, tendo uma relação positiva com os atributos $\mathrm{CBM}$ e C- $\mathrm{CO}_{2}$ (Quadro 3). Além de armazenador de nutrientes, o CBM é reportado como indicador rápido da interferência antrópica em Florestas de Araucária (Baretta et al., 2005). Por outro lado, a VCQ1 apresentou valores negativos de CCP para os atributos $\mathrm{K}, \mathrm{pH}\left(\mathrm{CaCl}_{2}\right), \mathrm{H}+\mathrm{Al}$ e $\mathrm{Mg}(-0,23$, $0,26,-0,53$ e $-0,69$, respectivamente). $\mathrm{O}$ sinal negativo de CCP nos atributos químicos indica que tais atributos são semelhantes nas áreas estudadas, sendo, portanto, considerados atributos supressores.

O elevado valor de correlação canônica entre atributos microbianos e químicos (Figura 2) ocorreu principalmente pelos maiores valores de $\mathrm{CC}$ dos atributos microbianos $\mathrm{CBM}$ e C- $\mathrm{CO}_{2}(0,87$ e 0,79, respectivamente) e químicos $\mathrm{COT}, \mathrm{P}, \mathrm{H}+\mathrm{Al}$ e $\mathrm{Ca}(0,78$, $0,74,0,71$ e 0,33 , respectivamente). Assim sendo, verificou-se maior sensibilidade dos atributos $\mathrm{CBM}$ e C- $\mathrm{CO}_{2}$ às variações dos atributos químicos $\mathrm{COT}, \mathrm{P}$, $\mathrm{H}+\mathrm{Al}$ e Ca nas áreas com araucária. Por outro lado, os atributos $\mathrm{pH}\left(\mathrm{CaCl}_{2}\right), \mathrm{K}$ e $\mathrm{Mg}(-0,57,0,03$ e 0,12, respectivamente) não influenciaram diretamente os atributos microbianos do solo, em virtude de seu baixo valor de CC. O efeito do $\mathrm{P}$ no crescimento da biomassa microbiana, bem como a relação positiva do Ca sobre o crescimento da biomassa, está demonstrado no trabalho de Tate et al. (1991). Maluche-Baretta et al. (2006), estudando o sistema convencional e orgânico de produção de maçãs em Urupema, SC, observaram por meio da ACC que o atributo microbiano CBM sofreu influência da concentração dos elementos $\mathrm{P}, \mathrm{Al}$ e COT no solo, e uma correlação negativa com $\mathrm{K}$, $\mathrm{Ca}$ e pH $\left(\mathrm{H}_{2} \mathrm{O}\right)$. Há evidências, também, de que os teores de $\mathrm{P}$ limitam a biomassa microbiana em algumas situações, mas não em outras, em que a adição de $\mathrm{P}$ pode exercer efeito benéfico (Biederbeck et al., 1984; Wardle \& Hungria, 1994).

A ACC mostrou ainda, por meio da análise de redundância na primeira correlação canônica, que 52 $\%$ da variação total dos atributos microbiológicos é explicada pela variação dos atributos químicos, enquanto apenas $36 \%$ da variação total dos atributos químicos é explicada pela variação dos atributos microbianos do solo das áreas com araucária. Esses resultados confirmam a maior sensibilidade dos atributos microbianos às variações químicas encontradas nas áreas estudadas e indicam que mudanças químicas pouco perceptíveis nos valores de COT, $\mathrm{P}$ e $\mathrm{Ca}$ no solo podem afetar atributos microbianos, especialmente o $\mathrm{CBM}, \mathrm{C}-\mathrm{CO}_{2}$ e suas relações no solo.

\section{CONCLUSÕES}

1. Os métodos de análise multivariada (ACD e ACC) são importantes ferramentas no estudo de indicadores de qualidade do solo, pois estabelecem relações entre os atributos químicos e microbianos e detectam diferenças entre as Florestas de Araucária. 
2. A análise de correlação canônica (ACC) mostrou que $52 \%$ da variação total dos atributos microbianos é explicada pela variação dos atributos químicos, e apenas $36 \%$ da variação total dos atributos químicos é explicada pelos atributos microbianos do solo das áreas com araucária.

3. A análise canônica discriminante (ACD) mostrou que a contribuição de cada atributo microbiano para a separação das áreas sofre variação ao longo do tempo e discriminou o $\mathrm{C}$ da biomassa microbiana (CBM) como sendo o atributo microbiano mais importante, seguido pela respiração basal $\left(\mathrm{C}-\mathrm{CO}_{2}\right)$.

4. Existem correlações significativas entre os atributos químicos e microbianos do solo, com destaque para o $\mathrm{CBM}$ e C- $\mathrm{CO}_{2}$, entre os atributos microbianos, e para COT e $\mathrm{P}$, entre os atributos químicos do solo.

\section{AGRADECIMENTOS}

Os autores agradecem ao $\mathrm{CNPq}$, pela bolsa concedida e ao programa BIOTA/FAPESP (www.biotasp.org.br), pelo financiamento dos projetos temáticos (processo $\mathrm{n}^{\circ}$. 01/05146-6, 01/05146-6), ao IBAMA, pela autorização de coleta (processo $\mathrm{n}^{0}$ 02027.002353/05-94). Aos funcionários da ESALQ (Denise Mescolotti, Dorival Grisotto e Luis Fernando Baldesin) e do Parque Estadual de Campos do Jordão, pelo auxílio durante as coletas.

\section{LITERATURA CITADA}

ALEF, K. \& NANNIPIERI, P., eds. Methods in applied soil microbiology and biochemistry. London, Academic Press, 1995. 576p.

ANDERSON, T.H. \& DOMSCH, K.H. The metobolic quotient for $\mathrm{CO}_{2}\left(q \mathrm{CO}_{2}\right)$ as a specific activity parameter to assess the effects of environment conditions, such as $\mathrm{pH}$, on the microbial biomass of forest soils. Soil Biol. Biochem., 25:393-395, 1993.

ANDERSON, T.H. Microbial eco-physiological indicators to assess soil quality. Agric. Ecosyst. Environ., 98:285-293, 2003.

ANDERSON, T.H. Physiological analysis of microbial communities in soil: Applications and limitations. In: RITZ, K.D. \& GILLER, K.E., eds. Beyond de biomass. London, British Society of Soil Science, 1994. p.67-76.

BARETTA, D. Fauna do solo e outros atributos edáficos como indicadores da qualidade ambiental em áreas com Araucaria Angustifolia no Estado de São Paulo. Piracicaba, Escola Superior de Agricultura Luiz de Queiroz, São Paulo, 2007. 158p. (Tese de Doutorado)
BARETTA, D.; BROWN, G.G.; JAMES, S.W. \& CARDOSO, E.J.B.N. Earthworm populations sampled using collection methods in Atlantic Forests with Araucaria angustifolia. Sci. Agric., 64:384-392, 2007.

BARETTA, D.; MAFRA, Á.L.; SANTOS, J.C.P.; AMARANTE, C.V.T. \& BERTOL, I. Análise multivariada da fauna edáfica em diferentes sistemas de preparo e cultivo do solo. Pesq. Agropec. Bras., 41:1675-1679, 2006.

BARETTA, D.; SANTOS, J.C.P.; FIGUEIREDO, S.R. \& KLAUBERG-FILHO, O. Efeito do monocultivo de pinus e da queima do campo nativo em atributos biológicos do solo no Planalto Sul Catarinense. R. Bras. Ci. Solo, 29:715724,2005 .

BIEDERBECK, V.O.; CAMPBELL, C.A. \& ZENTHEN, R.P. Effect of crop rotation and fertilization on some biological proprieties of a loam in Southwestern Saskatchewan. Canadian J. Soil Sci., 64:355-367, 1984.

INSTITUTO BRASILEIRO DO MEIO AMBIENTE E DOS RECURSOS NATURAIS RENOVÁVEIS - IBAMA. Lista oficial de espécies da flora brasileira ameaçadas de extinção. Diário Oficial da União, Brasília, 23 de Janeiro de 1992. p.870-872.

CHOROMANSKA, U. \& DELUCA, T.H. Prescribed fire alters the impact of wildfire on soil biochemical properties in a ponderosa pine forest. Soil Sci. Soc. Am. J., 65:232-238, 2001.

CRUZ-CASTILLO, J.G.; GANESHANANDAM, S.; MACKAY, B.R.; LAWES, G.S.; LAWOKO, C.R.O.O. \& WOOLLEY, D.J. Applications of canonical discriminant analysis in horticultural research. HortScience, 29:1115-1119, 1994.

DUMONTET, S.; DINEL, H.; SCOPA, A.; MAZZATURA, A. \& SARACINO, A. Post-fire soil microbial biomass and nutrient content of a pine forest soil from a dunal Mediterranean environment. Soil Biol. Biochem., 28:1467$1475,1996$.

EMPRESA BRASILEIRA DE PESQUISA AGROPECUÁRIA EMBRAPA. Centro Nacional de Pesquisa de Solos. Sistema brasileiro de classificação de solos. Rio de Janeiro, 1999. 412p.

FRANCHINI, J.C.; CRISPINO, C.C.; SOUZA, R.A.; TORRES, E. \& HUNGRIA, M. Microbiological parameters as indicators of soil quality under various soil management and crop rotation systems in Southern Brazil. Soil Till. Res., 92:18-29, 2007.

GAMA-RODRIGUES, E.F. Biomassa microbiana e ciclagem de nutrientes. In: SANTOS, G.A. \& CAMARGO, F.A.O., eds. Fundamentos da matéria orgânica do soloecossistemas tropicais e subtropicais. Porto Alegre, Gênesis, 1999. p.227-244.

GAUCH Jr, H.G. Multivariate analysis in community ecology. New York, Cambridge University Press, 1982. 298p.

GELSOMINO, A.; BADALUCCO, L.; AMBROSOLI, R.; CRECCHIO, C.; PUGLISI, E. \& MELI, S.M. Changes in chemical and biological soil proprieties as induced by antropogenic disturbance: A case study of an agricultural soil under recurrent flooding by wastewaters. Soil Biol. Biochem., 38:2069-2080, 2006. 
HUECK, K. As florestas da América do Sul. São Paulo, Polígono, 1972. 466p.

MALUCHE-BARETTA, C.R.D.; AMARANTE, C.V.T. \& KLAUBERG-FILHO, O. Análise multivariada de atributos do solo em sistemas convencional e orgânico de produção de maças. Pesq. Agropec. Bras., 41:15311539,2006

MARIANI, L.; CHANG, S.X. \& KABZEMS, R. Effects of tree harvesting, forest floor removal, and compaction on soil microbial biomass, microbial respiration, and $\mathrm{N}$ availability in a boreal aspen forest in British Columbia. Soil Biol. Biochem., 38:1734-1744, 2006.

NEARY, D.G.; KLOPATEK, C.C.; DEBANO, L.F. \& FFOLLIOTT, P.F. Fire effects on belowground sustainability: A review and synthesis. For. Ecol. Manag., 122:51-71, 1999 .

RAIJ, B.van; QUAGGIO, J.A.; CANTARELLA, H. \& ANDRADE, J.C. Análise química para avaliação da fertilidade de solos tropicais. Campinas, Instituto Agronômico de Campinas, 2001. 285p.

REITZ, R.; KLEIN, R.M. \& REIS, A. Projeto madeira do Rio Grande do Sul. Itajaí, Herbário Barbosa Rodrigues, 1988. $527 \mathrm{p}$.
ROBIM, M.J.; PASTORE, J.A.; AGUIAR, O.T. \& BAITELLO, J.B. Flora arbóreo arbustiva e herbácea de Parque Estadual de Campos do Jordão (SP). R. Inst. Flor., 2:31-53, 1990.

SAS Institute. SAS User's guide: Statistics. Versão 8.2. 6.ed. Cary, 2002.

TATE, K.R.; ROSS, D.J.; RAMSAY, A.J. \& WHALE, K.N. Microbial biomass and bacteria in two pasture soils: An assessment of measurement procedures, temporal variations and the influence of $\mathrm{P}$ fertility status. Plant Soil, 132:233-241, 1991.

TIEDJE, J.M.; CHO, J.C.; MURRAY, A.; TREVES, D.; XIA, B. \& AHOU, J. Soil teeming with life: New frontiers for soil science. In: RESS, R.M.; BALL, B.C.; CAMPEBELL, C.D. \& WATSON, C.A., Orgs. Sustainable management of soil organic matter. Wallingford, CAB International, 2001. p.393-412.

VANCE, E.D.; BROOKS, P.C. \& JENKINSON, D.S. An extraction method for measuring soil microbial biomass C. Soil Biol. Biochem., 19:703-707, 1987.

WARDLE, D.A. \& HUNGRIA, M.A. A biomassa microbiana do solo e sua importância nos ecossistemas terrestres. In: ARAÚJO, R.S. \& HUNGRIA, M.A., eds. Microrganismos de importância agrícola. Brasília, Embrapa-SPI, 1994. p.193-216. 
